23 | 1999

L'enseignement de la langue et de la littérature

françaises dans la seconde moitié du XIXe siècle

\title{
Didactique sans méthode : Le Nouveau Morand
}

Valeria De Gregorio Cirillo

\section{(2) OpenEdition \\ 1 Journals}

Electronic version

URL: https://journals.openedition.org/dhfles/3059

DOI: $10.4000 /$ dhfles.3059

ISSN: 2221-4038

Publisher

Société Internationale pour l'Histoire du Français Langue Étrangère ou Seconde

Printed version

Date of publication: 1 June 1999

Number of pages: p.366-381

ISSN: 0992-7654

\section{Electronic reference}

Valeria De Gregorio Cirillo, "Didactique sans méthode : Le Nouveau Morand", Documents pour I'histoire du français langue étrangère ou seconde [Online], 23 | 1999, Online since 06 July 2015, connection on 10 March 2023. URL: http://journals.openedition.org/dhfles/3059 ; DOI: https://doi.org/10.4000/dhfles. 3059

This text was automatically generated on 10 March 2023.

All rights reserved 


\title{
Didactique sans méthode : Le Nouveau Morand
}

\author{
Valeria De Gregorio Cirillo
}

Le texte des Dialogues italiens-français ou Le Nouveau Morand ${ }^{1}$ adapté par Candido Ghiotti, objet de notre analyse, constitue le dernier avatar, vers la fin du XIX ${ }^{\mathrm{e}}$ siècle, d'un volume publié à Paris en 1827 par Charles Morand ${ }^{2}$ dont le succès, en France comme en Italie, fut consacré par une longue série de rééditions ${ }^{3}$. Destiné à " l'enseignement des langues étrangères ", cet ouvrage ne sera examiné ici qu'en ce qui concerne sa partie dialogique. Cette partie est précédée par des Exercices méthodiques de nomenclature (p. 9-31) - bien entendu dans les deux langues - présentés par champs sémantiques tels que : la religion, l'univers, les lois physiques,, les couleurs, le temps, les minéraux, les végétaux, les animaux, l'homme, son corps, sa vie, les sens, la médecine, les facultés de l'âme, les aliments, les vêtements, la ville et les habitations, les meubles et les " ustensiles de ménage, les divertissements, la campagne, les professions, les poids et mesures ». Ceux-ci constituent de toute évidence le bagage mnémonique préliminaire de base, car ils présentent les mots thématiques fondamentaux qui figureront par la suite dans les dialogues dont ils annoncent en quelque sorte l'ampleur. Pour mieux en faciliter la compréhension, l'auteur offre en outre à l'apprenant un Vocabulaire Géographique, rédigé par ordre alphabétique et nanti d'explications très succinctes ( $\mathrm{p}$. 32-40), ainsi qu'une Table Alphabétique des noms propres d'hommes et de femmes les plus usités (p. 41-44). Cette partie propédeutique s'achève par une vaste gamme d'Exercices préliminaires (p. 45-66). Ces exercices présentent de courts énoncés, parfois des unités minimales ou même de simples interjections qui constituent des exemples ante litteram d'actes de parole (pour prier, demander, offrir, consentir, accorder, approuver, remercier, refuser, s'excuser, ...).

2 Ainsi qu'il en va dans toutes les réimpressions des volumes de Ch. Morand et de l'édition de C. Ghiotti, le texte français figure toujours en regard et sur la même page que les dialogues italiens, avec la seule différence que les éditions françaises présentent le texte français sur la partie gauche de la feuille, tandis que les éditions italiennes font l'inverse ; cette disposition met en évidence la conviction méthodologique que 
l'apprenant doit nécessairement partir de sa langue maternelle pour parvenir à la maîtrise et à la réalisation des actes de parole,, dans le nouveau contexte lexical et culturel.

3 Aucun métalangage grammatical, aucune suggestion méthodologique n'accompagnent ce recueil de dialogues, qui constitue un inventaire d'expressions en même temps qu'un modèle de communication, ce qui suggère à notre avis que les auteurs ne se soucient point d'élaborer une réflexion sur la langue ou de structurer des préceptes normatifs plus ou moins hiérarchisés, tout préoccupés qu'ils sont d'offrir au contraire le plus large éventail possible de situations interactionnelles. Cela tient peut-être aussi au fait que $\mathrm{Ch}$. Morand et $\mathrm{C}$. Ghiotti étaient des grammairiens et donc le renvoi à leurs ouvrages théoriques devait être sous-entendu. Le premier en particulier s'est intéressé à des problèmes de phonétique et d'orthographe en publiant des textes aussi bien en France qu'en Italie ${ }^{4}$, alors que le second, fin lexicologue, a rédigé un dictionnaire bilingue français-italien, ouvrage de grand succès pendant la première moitié du vingtième siècle ${ }^{5}$, qui a connu de très nombreuses réimpressions et mises à jour. Il a publié par ailleurs des ouvrages de grammaire et des chrestomathies ${ }^{6}$.

Néanmoins, dans la première édition italienne des Dialogues ${ }^{7}$, Charles Morand expose brièvement ses réflexions et ses objectifs (Aux étudions, p. 4-7). Sa carrière trentenaire d'enseignant $^{8}$ lui avait permis de relever qu'il n'existait pas de recueil satisfaisant et exhaustif de dialogues : aucun, écrit-il, n'était en mesure d'offrir toutes les connaissances nécessaires, de tels ouvrages présentant parfois des expressions grossières et de "sottes et impertinentes plaisanteries ", des registres linguistiques approximatifs, des arguments banals, peu intéressants et souvent déconnectés de la réalité des us et coutumes des deux pays. Il se décide ainsi à publier sa propre collection élaborée et expérimentée lors de ses cours particuliers. Ch. Morand, qui ne croit pas mériter par la publication de son recueil le titre d'auteur, précise qu'il ne s'est jamais " fait scrupule de s'approprier tout ce qu'il a jugé convenable à son but dans les autres ouvrages, se contentant de rectifier, supprimer, amplifier au besoin les parties qui en paraissaient susceptibles » $(1832$, p. 6). Il est sûr par ailleurs du succès d'une telle entreprise car, comme il l'affirme dans sa Réfutation, « Il est depuis long-temps reconnu par l'universalité de l'Europe instruite, que le Français est, de toutes les langues vivantes, sans exception, la plus claire, la plus exacte, la plus précise, la plus apte enfin à communiquer la pensée » $(1829$, p. 6). Le français permet donc d'appréhender les grands chefs-d'œuvre littéraires, mais constitue aussi un intermédiaire politique incontournable ; en effet « le fameux Congrès a préféré le français aux langues des diplomates assemblés » (ibid., p. 7).

5 Son riche corpus, presque un inventaire d'occasions interactionnelles, semble bien représentatif d'une société élitaire, où s'impose la présence masculine aux prises avec des activités nobles (manège, escrime, chasse) ou d'agrément (collections, voyages, spectacles) et engagée dans des occupations ou des métiers utiles (militaires, artisans, commerçants). Au contraire, la présence des femmes et leur sphère d'influence est circonscrite au simple cercle familial, raison pour laquelle le nombre des dialogues où elles interviennent est fort réduit ${ }^{9}$.

6 Eu égard aux destinataires du recueil et aux intentions des auteurs, les registres de langue sont toujours soignés, car la lecture et la mémorisation des séquences devraient permettre aux apprenants de parvenir à une expression orale autonome, qui reflète néanmoins le modèle proposé. Le paradoxe foncier de toutes ces formes discursives 
inventoriées pour l'apprentissage des langues consiste précisément dans le fait que l'élaboration linguistique intentionnellement non connotée et la rigide modélisation planifiée d'un produit clos ne peuvent aucunement garantir la spontanéité individuelle et l'expression contextualisée d'actes langagiers.

7 Il faut aussi supposer que les Dialogues, dans la mesure où ils n'étaient pas liés à un cours d'études statutaires et où leur destination n'était pas exclusivement réservée aux classes d'enseignement, pouvaient être utilisés par les auditeurs les plus hétérogènes. Ainsi, à côté de textes édifiants qui offrent des leçons de morale à bon marché, il y a aussi de courtes scènes qui frisent le comique du non-sens, comme par exemple le Dialogue Ll - Entre un grand seigneur et un cocher. Ici la personne qui offre ses services pour être employée comme cocher n'a aucune expérience de la conduite d'une voiture, mais pense pouvoir pallier cette carence par un savoir, selon ses dires, essentiel pour un cocher « tout formé ». " Mais, Monseigneur, je le suis ; je sais sur le bout du doigt le répertoire de toutes les injures qu'il faut dire à un charretier, à un cocher de fiacre qui oserait barrer ou retarder ma marche, à un cocher bourgeois qui voudrait me croiser ou me gagner de vitesse. Je n'ignore aucun des propos durs et insolents qu'il faut tenir à un piéton qui ne se range pas assez vite ; en un mot, je saurais tout, si je savais conduire la voiture ; mais je l'aurai bientôt appris». Il nous reste à nous interroger sur l'exploitation possible dans la vie réelle d'une telle saynète : à qui aurait-elle bien pu servir de modèle?

De même, le dialogue LIX - Le mérite de l'homme met en scène avec un vif humour une anecdote sur l'hypocrisie sociale. L'un des deux locuteurs a reçu l'homme qui lui a rendu visite avec beaucoup de froideur à cause de sa tenue vestimentaire peu soignée, mais l'autre locuteur lui apprend qu'il s'agit d'un comte : «- Que dites-vous ? Comte ! Ciel, que ne l'ai-je su ! je vais lui envoyer mon domestique pour me faire excuser de mon mieux. Où demeure-t-il ? -Dans son palais. - Quoi ! il a un palais ? Est-il donc si riche ? - Il a cent mille écus de rente. - Vous me mettez au désespoir. Je cours chez lui me jeter à ses pieds ».

9 Malgré la brièveté des relations qui s'instaurent entre les interlocuteurs rassemblés selon les exigences des énoncés, parfois ceux-ci ne manquent pas d'une certaine épaisseur psychologique et partant de quelques réflexions exemplaires. La forme dialogique, qu'on pourrait définir pragmatique, choisie par Morand, n'a d'ailleurs rien de particulièrement original : dès les premières parutions de grammaires à l'usage des étrangers, une partie plus ou moins étendue y était consacrée à l'interaction verbale avec des prototypes dialogiques recouvrant le plus grand nombre possible de réalisations discursives ayant trait à la vie familiale et sociale. Mais le modèle créé par $\mathrm{Ch}$. Morand constitue une réussite durable : nous en avons la preuve tangible non seulement dans le grand nombre de ses éditions, mais aussi dans le fait que des grammaires traditionnelles de l'époque, comme par exemple celle de Ludovico Goudar, dès l'édition de $1834^{10}$, propose environ une vingtaine de dialogues familiers, sélectionnés un peu au hasard. De même, ces dialogues sont repris dans des manuels scolaires de morceaux choisis ${ }^{11}$; le recours à la forme dialoguée s'avère donc un instrument attrayant au service de la pédagogie.

Pour revenir à notre texte, $\mathrm{C}$. Ghiotti, avec un souci évident mais tout aussi ambigu de mise à jour, intitule l'ouvrage dont il est l'éditeur, vu le temps écoulé depuis la parution de l'original, Le Nouveau Morand, et insiste dans le sous-titre sur son double apport en matière d'adaptation et d'enrichissement. Il affirme en effet assez emphatiquement que 
les modifications apportées aux dialogues concernent les formes et les sujets de la moderne conversation, reproposant ainsi, mais sûrement sans s'en rendre compte, le problème terminologique de conversation $v s$ dialogue. D'un côté, l'enrichissement qu'il proclame est tout à fait fictif car les exercices préliminaires de nomenclature méthodique et de phraséologie, ainsi que le vocabulaire géographique et des noms propres, sont déjà présents, comme nous l'avons vu, dans la toute première édition française; de l'autre, les adaptations proposées apparaissent également décevantes. Regardons-y de plus près.

11 Ce n'est que dans un tout petit Nota bene au bas de la table des matières; (p. 321) que Fauteur attire l'attention sur la portée véritable de ses interventions; il déclare donc que les dialogues marqués d'un astérisque sont, tout à fait nouveaux, que ceux avec deux astérisques « ont subi des modifications radicales » et qu'enfin « tous les autres ont été, sans exception, améliorés ". Étant donné que C. Ghiotti ne nous donne aucun renseignement sur le texte d'origine et qu'il est donc impossible d'établir une confrontation digne de foi, nous avons décidé de comparer d'une manière suivie son texte avec la dernière édition française de $1854^{12}$, d'une part et, de l'autre, avec l'édition italienne de $1868^{13}$, celle-ci étant apparemment la' dernière dans le cursus éditorial de ces dialogues. Dès le début il est apparu évident que l'édition de 1868 n'apportait que de rares enrichissements à l'édition de 1854, tout en gardant la même progression, et qu'il était donc inutile de prendre en considération la plus ancienne : il suffisait de se cantonner à examiner le texte de C. Ghiotti de 1877 par rapport à l'édition de Turin, car certaines présences-absences permettaient d'affirmer que l'auteur n'avait tenu en aucun compte l'édition française. Procédant en effet à une mise en parallèle des tables des dialogues des éditions de 1854 et de 1868 (124 dialogues dans la première et 127 dans la deuxième) nous avons pu relever que la progression numérique y est identique jusqu'au n. XLI ; le dialogue XLII - Sur les théâtres, manque en effet dans l'édition de 1868. L'introduction cependant d'un dialogue nouveau, XLIV - De la' loterie, rétablit la succession, qui s'achève avec le dialogue LIV - Une église. Après celui-ci, la numérotation est quelque peu modifiée par l'introduction de cinq nouveaux dialogues : LV - D'un mariage, LXXXIV - Entre un recruteur et un journalier, CXII - Une auberge en Espagne, CXXVI - Une couturière, CXXVII - Un marchand de tableaux, et par la suppression de deux dialogues figurant dans l'édition de 1854, respectivement : CXXII - L'or et l'éloquence, et CXXIV - L'opulence et la pauvreté.

12 En prenant donc le départ de cette édition de 1868 , il nous est possible de vérifier la portée exacte des interventions de C. Ghiotti. Il faut tout d'abord remarquer que celuici a entièrement bouleversé l'ordre des dialogues, vraisemblablement dans le dessein d'aboutir à une progression plus cohérente des thèmes proposés. Selon la déclaration détaillée de l'auteur, les dialogues nouveaux seraient au total neuf et les dialogues radicalement modifiés treize ${ }^{14}$. En ce qui concerne le premier groupe, il est nécessaire de remarquer : 1) que le dialogue XXXI - Voyage en bateau à vapeur, englobe, avec quelques menues variations, les douze répliques du dialogue XXXIX - Pour s'embarquer $(1868$, p.79) ; 2), que le dialogue CXVIII - De l'astronomie, de la géologie et de la physique, correspond, malgré divers ajouts et l'intégration d'une dernière partie concernant l'électricité, au dialogue XC - De l'astronomie et de la physique (1868, p. 190) ; 3), que le dialogue CXX - Des mines, utilise quand même les toutes premières répliques du dialogue LXXXV du même titre $(1868$, p. 179) ; 4) que le dialogue CXXIII - De la construction des vaisseaux et des machines à vapeur, reprend le dialogue LXXX - De la construction des vaisseaux (1868, p. 164), en l'amplifiant toutefois par nombre de détails. 
Les cinq autres dialogues sont effectivement inédits et certains se rapportent à des situations spécifiques et nouvelles de la vie moderne.

L'apport de C. Ghiotti est ainsi ramené à ses justes proportions, et si nous pouvons affirmer que dans l'ensemble la plupart des énoncés n'ont pas subi la moindre retouche, il nous reste à quantifier les " modifications radicales " revendiquées par l'auteur. Dans un souci évident d'actualiser un tant soit peu les répliques (le laps de temps de cinquante ans séparant les deux éditions nous apparaît aujourd'hui inconcevable), Ghiotti a dû se poser le problème que les conditions socio-politiques ambiantes avaient bien évolué, même si le public était prêt à accepter comme au goût du jour en 1877 le langage et le style de 1827 et sans doute plus éloignés encore dans le temps si nous devons en croire les affirmations de Morand. C'est donc surtout dans ce sens et sur des détails que l'auteur a travaillé pour améliorer et parfaire sa rédaction. Il a d'abord rectifié certains titres dont le libellé pouvait apparaître désuet, tels que $L a$ chassomanie (204) ${ }^{15}$ qui devient Sur la manie de la chasse (231) ; Les Gazettes (196), Les journaux (236) ; Du métier des armes (171), Du service militaire (242). Il a ensuite modernisé toutes les unités de mesure. D'abord les unités de longueur : les aunes (« il vous en faudra une aune trois quarts ", 62) deviennent mètres (88) ; une lieue (" on compte une lieue d'une extrémité à l'autre », 78) devient " deux milles » (124); " trois pieds six pouces » (137) deviennent « un mètre et demi » (142) et « deux lieues de circonférence " (82) « huit kilomètres " (128). Même chose pour ce qui concerne les mesures de superficie : « cinq cents arpens » (82) deviennent « cinq cents hectares » (128), et pour les capacités : « deux cordes de bois de hêtre » (90) deviennent « six stères » (97); « une feuillette [de vin] » (47) devient «trois hectolitres » (96) et « un quartaut » (47) « une pièce de deux cents litres» (96). Quant aux unités monétaires et aux changements de leurs valeurs, on a l'impression que l'auteur progresse de façon quelque peu ondoyante, peut-être à cause du nombre considérable de renvois au système économique contemporain, ainsi " un louis, vingt-quatre francs " (c'est le prix d'un chapeau, 53), correspond à «quinze francs » (86), « un écu de six francs » (mis pour jouer à la loterie, 86) devient simplement " un écu " (170), " cent écus et un louis d'étrennes " pour la femme de chambre (61) deviennent «trois cents francs par an » (73) ; un louis (« auriez-vous la monnaie d'un louis ? » 42) devient « une pièce de vingt francs » (85); « il y manque l'appoint d'un sequin » (69) devient « il y manque précisément une pièce de vingt francs " (104) et "s'il me paye en ducats de Hollande non rognés et de poids " (67) devient, en termes plus généraux, « s'il me paye en or » (103).

14 L'auteur propose aussi certaines corrections compte tenu de la modification de la qualité de l'environnement urbain. Ainsi « as-tu décrotté mes souliers ?»(37) devient « as-tu ciré mes bottines ?» (81) et " monte dans le carrosse » (50) « monte en voiture » (84). Il faut bien tenir compte, par ailleurs, qu'en matière d'habillement aussi la mode a changé; ainsi « la ganse d'or et le petit velours » (53) qui ornaient un chapeau en 1868 laissent la place à « un ruban noir et un petit galon de soie » (86); la blonde (« il me faut une robe de blonde à volant ", 265) devient le tulle (154) ; "un corsage à la grecque " (265) devient " un corsage décolleté » (154); « la mousseline de Naples moirée » (266) devient « la mousseline laine » (154), tout en insistant avec les expressions « à la mode " ou « au dernier goût », qui accompagnent les mêmes ornements.

15 Fait curieux pour un spécialiste de français, Ghiotti transforme quelques renvois au contexte culturel français - qu'il s'agisse de toponymes ou de références à la littérature et à l'histoire - en les adaptant au milieu italien ainsi « un Télémaque » (145) devient « 
un Dante » (94), « les sermons à Bourdaloue » (146) « Les Fiancés de Manzoni » (95), « la Rue d'Enfer » (49) « Rue du Po » (84), « la révolution de France » (224) « les vicissitudes des politiques d'Italie » (262), « le vin de Bourgogne » (47) « le vin d'Asti »(96).

L'inventaire de ces exemples met en évidence qu'il n'y a pas de véritables transformations de fond et que les rapports entre individus, ainsi que les échanges « inégalitaires » entre maitres et valets (ou femmes) de chambre, perruquiers, cochers et négociants demeurent immuables, la modernisation n'étant pour Ghiotti qu'un fait terminologique ou le résultat contingent de certains progrès techniques. Ainsi, dans le dialogue LXXXV11 -De la campagne, le riche propriétaire achève-t-il son discours sur le battage du blé en déclarant : "l'année prochaine je compte m'élever au niveau du progrès. On emploie de nos jours des machines à battre qui font à elles seules, en un jour, le travail de tous mes batteurs réunis » $(2 \mathrm{2I})^{16}$.

17 En fait, le nombre des dialogues où l'intervention de Ghiotti se révèle appréciable est plutôt restreint : nous signalons en particulier Du service militaire, La guerre etla victoire et la dernière partie de Pour voir la ville. Il en découle que Ghiotti reste forcément ancré dans un monde et dans des usages révolus et qu'il ne modifie en rien certains tours de parole : par exemple sur la façon de faire le café ${ }^{17}$ ou sur le travail du perruquier ${ }^{18}$; mais si l'actualité le requiert, il veille cependant à mieux nuancer. C'est ainsi que, si dansle dialogue XXVI - Du commerce, il reprend inchangées les affirmations «J'aibeaucoup perdu pendant la révolution » (99) et « Les riches capitalistes, quiont des fonds à placer, gagnent beaucoup dans ce moment» (99),par lasuite il modernise la remarque sur le papier monnaie ${ }^{19}$.

18 Dans tous les dialogues, de longueur variable, les participants (deux en général) alternent dans les prises de parole, qui peuvent être plus ou moins longues (d'une ligne à un maximum de vingt-cinq). Le démarrage de la conversation met en évidence qu'il s'agit presque toujours de rencontres occasionnelles dans des situations plausibles aptes à faire aborder n'importe quel sujet, qui pourra être plus ou moins développé par la suite. L'espace où les personnages des dylogues se déplacent n'est jamais explicité, mais l'échange d'ouverture permet de le déterminer ; de même, celle-ci laisse entendre si la situation est familière ou formelle. Peu nombreuses sont les informations extralinguistiques concernant les gestes, les attitudes, les jeux de physionomie entre locuteurs et allocutaires ${ }^{20}$.

19 L'élaboration d'un corpus écrit qui ne vise que la seule communication orale devait nécessairement présenter une gamme étendue d'actes illocutoires comportant un vaste éventail d'énoncés (déclaratifs, performatifs, interrogatifs, impératifs, exclamatifs). Dans les interventions initiales, les tournures sont presque toujours interrogatives ou impératives, mais on rencontre aussi des expressions phatiques introduites par des morphèmes interjectifs à valeur argumentative.

Nous conclurons donc en nous interrogeant sur la validité du projet de Ghiotti : probablement attiré par le prestige d'une tradition indiscutable ou par simple calcul éditorial, il a cru opportun de proposer, à l'aube du vingtième siècle, un texte dans lequel pointent encore de subtiles atmosphères 'ancien régime'. Il reste qu'après cette date les dialogues de Morand continuent d'être édités pour ne disparaître du marché éditorial qu'après 1923, sans doute parce qu'expressions et contenus ne sont plus susceptibles de remaniements ultérieurs, mais surtout pour des raisons extralinguistiques : les nouvelles générations ne portent plus aucun intérêt à la décodification de messages dont la teneur leur semble à des années-lumière de leur 
réalité. Ghiotti, ainsi que ses contemporains, ont sous-estimé le fait que le dialogue, inscrit dans un espace-temps mouvant, nécessite d'un dynamisme incessant qui fait défaut à sa perspective somme toute conservatrice. Sa stratégie pédagogique, qui a certainement eu une retombée concrète à son époque, n'a pu soutenir l'impact des nouvelles méthodologies de la didactique des langues.

\section{BIBLIOGRAPHY}

FERRANTE,. Ferdmando (1853) : Pezzi scelti di autori francesi ad uso delle scuole D.C.D.G., Palermo, Tip. Clamis e Roberti (deuxième: édition, 1859).

GHIOTTI, Candido (1868) : Grammatica ragionata della lingua francese. Nuovo corso di lezioni teoricopratiche dettate dal confronto della lingua italiana e coll 'analisi filologica, Alessandria, Ragazzone. GHIOTTI, Candido (1877) : Dialogues italiens-français adaptés-aux formeset aux sujets de la moderne conversation et enrichis d'exercices préliminaires de nomenclature méthodique et de phraséologie vocabulaire Géographique et de Noms propres de personnes. Turin Favale.

GHIOTTI, Candido (1883) : Nuovo dizionario comparativo delle lingue italiana e francese, Torino, Bellardi e Appiotti.

GHIOTTI, Candido (1886) : La nomenclature en action. Exercices élémentaires de conversation précédés d'un traité de prononciation à l'usage des élèves des écoles techniques, Turin, Petrini.

GHIOTTI, Candido (1887) : Leçons françaises de littérature et de morale. Choix de morceaux en prose et en vers, extraits desmeilleurs écrivains, Turin, Paravia.

GOUDAR, Ludovico (1834) : Nuova grammatica italiana con nuove regole e spiegazioni intorno la moderna pronunzia de' dittonghi francesi, ricavate dllte opere de' migliori grammatici.

Nuovissima edizione arricchita per la prima volta di terni o d'esercizi di sintassi e dei dialoghi famigliari di C. Morand, Livorno, Tipografia Vignozzi.

LILLO, Jacqueline (1990) : Les grammaires de Ludovico Goudar (1744-1925), Quaderno n³0, Université di Palermo, Stass.

MADIA, Giuseppe (1857) : Morceaux choisis de lectures françaises avec des synonymes appliqués aux mots les plus usités ; à l'usage de tous les établissements littéraires tant nationaux qu'étrangers, Naples, Imprimerie Ruelle St.-Sévère.

MINERVA, Nadia / Pellandra, Carla (1997) : Insegnare il francese in Italia--Repertorio analitico di manuali pubblicati dal 1625 al 1860, Bologna, CLUEB.

MORAND, Charles (1811) : Nuovo metodo di lettura francese coll'ajuto della pronuncia italiana, Milano, Gio. Bernardoni.

MORAND, Charles (1815) : Trattato generale della pronuncia francese ossia dizionario grammatico-logicocritico di tutte le voci della lingua, Milano, Gio. Pirotta.

MORAND, Charles (1823) : Esercizj sulle conjugazioni francesi e quadro intero di tutti i verbi, nella libreria dell'Autore, Milano. 
MORAND, Charles (1827) : Dialogues classiques, familiers et autres, à l'usage des étudions des langues française et italienne, avec des exercices préliminaires, édition confiée aux soins de l'abbé Lauri, Paris, Cormon et Blanc.

MORAND, Charles (1829) : Réfutation de la réforme orthographique, chez l'auteur, grand-rue SteCatherine, Lyon.

MORAND, Charles (1831) : Essai d'un traité général et analytique de la prononciation française, ou Dictionnaire de toutes les solutions nécessaires sur les difficultés du langage...., Paris, Cormon et Blanc. MORAND, Charles (1832) : Dialogues classiques, familiers et autres à l'usage des étudians des langues italienne et française, Livoume, del'Imprimerie Le Phénix.

MORAND, Charles (1854) : Dialogues classiques, familiers et autres, à l'usage des étudiants des langues française et italienne, avec des exercices préliminaires, septième édition, soigneusement revue et corrigée par S.-H. Blanc et Frignani, Paris et Lyon, Ancienne Maison Cormonet Blanc, S.-H. Blanc et Cie.

MORAND, Charles (1868) : Dialogues français-italiens précédés d'exercices préliminaires et suivis d'un vocabulaire géographique etd'unrecueil de noms propres, nouvelle édition, Turin, C. Favale et Comp.

\section{NOTES}

1. Dialogues italiens-français adaptés aux formes et aux sujets de M moderne conversation et enrichis d'exercices préliminaires de nomenclature méthodique et de phraséologie, d'un vocabulaire Géographique et de Noms propres de personnes par C. Ghiotti, Professore nella Regia Scuola Tecnica Monviso, Turin, Favale, 1877. La toute dernière édition, au tournant du siècle, publiée en 1899 par l'éditeur Paravia ( $\mathrm{T} \backslash$ irin-Rome-Milan-Florence-Naples), est identique à celle-ci, la seule différence étant dans le titre de C. Ghiotti, actuellement : " Direttore della Regia Scuola tecnica Sommeiller ». Même si dans les textes des Dialogues analysés les frontispices sont dans les deux langues, nous avons choisi de ne mentionner que la version française.

2. Dialogues classiques, familiers et autres, à l'usage des étudians des langues française et italienne, avec des exercices préliminaires par $\mathrm{Ch}$. Morand, Edition confiée aux soins de l'abbé Lauri, Paris, B. Cormon et Blanc, 1827. En cette même année et chez le même éditeur, Ch. Morand fait paraître un texte français-espagnol, identique au précédent. Une deuxième édition des Dialogues est imprimée à Paris en 1833 chez Cormon et Blanc, « confiée aux soins du professeur Sforzosi ».

3. À titre indicatif, les éditions des Dialogues de Ch. Morand sont environ une quarantaine, imprimées dans différentes villes italiennes (Livourne, Milan, Venise, Naples, Turin) ; les éditeurs aussi changent : outre l'abbé Lauri et Blanc et Frignani, rappelés dans les notes, nous pouvons citer aussi les noms de Luigi Sforzosi, Charles Saint-Ange de Virgile, C.G. Borgstrom, Giuseppe Madia, Luigi Dupin. En ce qui concerne la réédition par C. Ghiotti, les publications sont environ une vingtaine. Il nous a semblé inutile de les répertorier dans le détail.

4. Ch. Morand, Nuovo metodo di lettura francese coll' ajuto della pronuncia italiana, Milano, Gio. Bemardoni, 1811 ; Trattato generale della pronuncia francese ossia dizionario grammatico-logico-critico di tutte le voci della lingua, Milano, Gio. Pirotta, 1815 ; Esercizi suite conjugazioni francesi e quadro intero di tutti $i$ verbi, nella libreria del l'Autore, Milano, MDCCCXXIII ; Réfutation de la réforme orthographique par Ch. Morand de Lyon, ancien professeur de langues, d'histoire, de géographie, etc., chez l'auteur, grand-rue Ste-Catherine, n. 9, Lyon, 1829 ; Essai d'un traité général et analytique de la prononciation française, ou Dictionnaire de toutes les solutions nécessaires sur les difficultés du langage parlé, divisé en quatre parties, contenant : $1^{\circ}$ Élémens des sons, des articulations; mécanisme et tableau des différentes sortes de syllabes ; $2^{\circ}$ Variétés des voyelles, et tables complètes des diphtongues ; $3^{\circ}$ 
Consonnes et leurs irrégularités dans tous les cas, tables des $H$ aspirées, des L doubles et mouillées ; $4^{\circ}$ Règles de la prosodie, et tables des longues, brèves et douteuses. On y joint : de nombreuses observations sur l'harmonie, les beautés de cette partie de la langue, et une esquisse des principes de l'histoire naturelle, servant de modèles d'analyse, au moyen de laquelle on parvient graduellement à exécuter soi-même la peinture de la parole, acquérir la rectitude raisonnée de l'orthographe et de la prononciation, Paris, Cormon et Blanc, 1831. Ainsi que l'auteur le souligne lui-même dans Y Introduction, le texte reprend la configuration des deux livres publiés à Milan « destinés à faciliter aux Italiens l'intelligence de notre prononciation » (p. 10). Selon Ch. Morand l'étude des langues vivantes compte trois progressions : parler, lire, écrire : «L'enfant apprend à parler, l'adolescent à lire, le jeune homme à écrire » (p. 5). Malheureusement, la première éducation étant presque toujours « concentrée entre les mains des femmes, la plupart illettrées », il se doit d'offrir les moyens pour « acquérir la pureté du langage » et « rendre populaire la bonne prononciation » (p. 8).

5. Nuovo dizionario comparativo delle lingue italiana e francese, Torino, Bellardi e Appiotti, 1883, $2 \mathrm{v}$.

6. Nous ne citons que les premières éditions : Grammatica ragionata della lingua francese. Nuovo corso di lezioni teorico-pratiche dettate dal confronto della lingua italiana e coll'analisi filologica, Alessandria, Ragazzone, 1868 ; La nomenclature en action. Exercices élémentaires de conversation précédés d'un traité de prononciation à l'usage des élèves des écoles techniques, Turin, Petrini, 1886 et Leçons françaises de littérature et de morale. Choix de morceaux en prose et en vers, extraits des meilleurs écrivains, Turin, Paravia, 1887.

7. Dialogues classiques, familiers et autres à l'usage des étudians des langues italienne et française, Livourne, de l'Imprimerie Le Phénix, 1832

8. Dans sa Réfutation ... de 1829 il déclare être le « créateur d'un mode d'enseignement [qu'il] professe depuis près de quarante ans » (p.3).

9. Une réplique (Entre un gentilhomme et son épouse sur la manie de la chassé) synthétise bien la disparité entre les deux sexes : " la nature a donné aux femmes un cœur tendre et compatissant ; aux hommes la valeur et le courage » (p. 233), et souligne ailleurs la crédulité et les préjugés féminins ( Je suis peiné de voir qu'une femme d'esprit comme toi, soit si faible. Tu devrais rougir de ta crédulité », p. 265).

10. L. Goudar, Nuova grammatica italiana e francese, con nuove regole e spiegazioni intorno la moderna pronunzia e la natura de ' dittongh i franc e si, ricavate dalle opere de' migliori grammatici. Nuovissima edizione arricchita per la prima volta di tenu o d'esercizi di sintassi e dei dialoghi famigliari di C. Morand, Livorno, Tipografia Vignozzi, 1834. En ce qui concerne les très nombreuses éditions successives, nous renvoyons à J. Lillo, Les grammaires de Ludovico Goudar (1744-1925), Quaderno n³0, Université di Palermo, Stass, 1990, ainsi qu'au répertoire édité par N. Minerva et C. Pellandra, Insegnare il francese in Ilalia - Repertorio analitico di manuali pubblicati dal 1625 al 1860, Bologna, CLUEB, 1997.

11. Ferdinando Ferrante, Pezzi scelîi di aulori francesi ad uso delle scuole D.C.D.G. Palermo, Tip. Clamis e Roberti, 1853 (deuxième édition, 1859), et Giuseppe Madia, Morceaux choisis de lectures françaises avec des synonymes appliqués aux mots les plus usités; à l'usage de tous les établissements littéraires tant nationaux qu'étrangers, Naples, Imprimerie Ruelle St.-Sévère, 1857.

12. Dialogues classiques, familiers et autres, à l'usage des étudiants des langues française et italienne, avec des exercices préliminaires, septième édition, soigneusement revue et corrigée par S.-H. Blanc et Frignani, Paris et Lyon, Ancienne Maison Cormon et Blanc, S.-H. Blanc et Cie, 1854.

13. Dialogues français-italiens précédés d'exercices préliminaires et suivis d'un vocabulaire géographique et d'un recueil de noms propres, nouvelle édition, Turin, C. Favale et Comp., 1868.

14. Plus précisément les premiers seraient : XXXI - Voyage en bateau à vapeur, XXXVII

- Voyage en chemin de fer, XLI - En omnibus, XLIII - À la poste, LXXVIII - Au théâtre, CXVIII - De l'astronomie, de la géologie et de la physique, CXIX - Au bureau des télégraphes, CXX - Des mines, CXXIII De la construction des vaisseaux et des machines à vapeur et les deuxièmes : XVI - Avec un chapelier, XIX -Avec le tailleur, XXIII - Avec un marchand de vin, XXVI - Du commerce, XXXVI 
- En arrivant dans un hôtel, XXXIX - Pour voir la ville, XLII - De l'écriture et de ses accessoires, LVI - Avec une couturière, marchande de modes, LXXXVII - De la campagne, CIII - Entre un recruteur et un journalier, CIV - Du service militaire, CVI - Après une bataille (entre deux officiers supérieurs), CXXI - De la typographie. Pour tous ces dialogues, bien que le titre en italien précède le titre français, nous n'indiquons que ce dernier. Pareillement, nous avons décidé de ne pas analyser les traductions. Il nous paraît toutefois intéressant de rapporter la dernière réflexion de $\mathrm{Ch}$. Morand dans son avis Aux étudians : «Je dois ajouter encore, que je professe la maxime invariable de ne jamais rien faire en italien sans consulter les professeurs les plus accrédités de cette langue; d'où il suit que, si mes faibles lumières ne m'ont pas égaré dans la mienne propre, j'oserais me flatter qu'on n'a point encore produit, entre l'italien et le français, une correspondance aussi exacte que celle que je me suis efforcé d'établir dans ces Dialogues » $(1832$, p. 6). Les dialogues présentés par Ghiotti sont au total 132, ils étaient 127 dans l'édition de 1868.

15. Les chiffres entre parenthèses en gras renvoient aux pages de l'édition de 1868 , les autres à celle de 1877.

16. Voir aussi le terme « vacciner » (265) à la place de « inoculer » (208), ou " mettrebas » (206) au lieu de « pouliner» (156).

17. «- Comment le faites-vous ? - À l'ancienne méthode : je mets tout uniment lecafémoulu dans l'eau bouillante, j'éloigne la cafetière du feu, je la laisse reposer, et leclarifie ensuite avec de la colle de poisson; après quoi, je tire au clair et je jettele marc » (77).

18. «[...] je compose une perruque, dont je choisis le modèle parmi les portraits des personnages illustres de l'histoire moderne, ou de l'antiquité. C'est ainsi que jeforme des guerriers, des sages et des héros » (83).

19. «Les changeurs, par exemple, font de grands profits ; il n'y a plus que du papier--monnaie!» (99) au lieu de "C'était dans le temps du papier monnaie qu'on pouvait faire de grands profits » (64). Reste identique à elle-même, par contre, une réflexion sur la crise du commerce des soieries : « [...] le moment actuel est peu favorableà ce genre de commerce ; on n'a plus les mêmes débits qu'autrefois», et les plus bellesétoffes se donnent aujourd'hui à vingt pour cent au dessous du prix de fabrique » (107).

20. Quelques indices de nature non verbale se trouvent dans les dialogues LV - Conversation entre deux demoiselles, XCII - La bonne fête (Entre le maître et son jardinier), CXXX - La Grâce et la Beauté.

\section{AUTHOR}

\section{VALERIA DE GREGORIO CIRILLO}

Istituto UniversitarioOrientale,Napoli 